\title{
Teleradiology via Narrow-Band Integrated Services Digita I Network (N-ISDN) and Joint Photographic Experts Group (JPEG) Image Compression
}

\author{
G. James Blaine, Stephen M. Moore, Jerome R. Cox, and Robert A. Whitman
}

\begin{abstract}
The importance of remote access to both radiological images and medical information has stimulated many demonstration projects that use a variety of telecommunications providers' offerings. Teleradiology, through modest cost channels, can achieve adequate response times using a combination of narrow-band integrated services digital network (N-ISDN) and data compression. A demonstration project, developed in collaboration with Southwestern Bell Technology Resources, Inc, uses the aggregate bandwidth of two B channels (achieving a rate of $\mathbf{1 2 0}$ kilobits per second) and a block-oriented discrete cosine transform compression/decompression implementation based on the Joint Photographic Experts Group Standard for Still Image Compression. System response measurements for an Inquiry and Display Station accessing the Mallinckrodt Institute of Radiology's Radiology Image and Information Management Testbed via the N-ISDN connection show response times to be within 20 seconds. Viewing applications have been shown at sites within St Louis and at Radiological Society of North America, 1990, in Chicago.
\end{abstract}

KEY WORDS: teleradiology, compression, Joint Photographic Experts Group, narrow-band integrated services digital network.

\begin{abstract}
A LTHOUGH THE PROMISED widespread availability and low cost of basic-rate (or narrow-band) integrated services digital network (N-ISDN) channels have not yet been achieved, recent events suggest a break in the "gridlock" between the Regional Bell Holding Companies (RBHCs), the switch equipment manufacturers, and the customer premise equipment vendors. ${ }^{1}$ Issues related to investment, interoperability, connectivity, and pricing are
\end{abstract}

From the Mallinckrodt Institute of Radiology, Washington University Medical School, and the Department of Computer Science, Washington University Engineering School, Washington University, St Louis, MO.

Supported by Southwestern Bell Technology Resources, Inc, and Southwestem Bell Telephone Company, St Louis, MO.

Address reprint requests to G. James Blaine, DSc, Mallinckrodt Institute of Radiology, $510 \mathrm{~S}$ Kingshighway Blvd, Box 8131, St Louis, MO 63110.

Reprinted with permission from Medical Imaging V7: Picture Archiving and Communications Systems, Society of PhotoOptical Instrumentation Engineers, 1992.

0897-1889/92/0503-0010\$03.00/0 being resolved. Deployment of facilities equipment for five of six surveyed major RBHCs indicate availability (not necessarily deployed) will be in the range of $50 \%$ to $90 \%$ of total lines by $1994 .^{2}$ The Transcontinental ISDN Project $1992^{3}$ should produce a focus for resolving the needed interoperability. Although our research and development programs at Washington University have an intense focus on high-bandwidth local-area and wide-area facilities, ${ }^{4}$ we recognize the potential opportunity to support telemedicine applications for the primary care physician via $\mathrm{N}$-ISDN facilities and $\mathrm{N} \times 56$ kilobits per second $(\mathrm{kb} / \mathrm{s})$ service..$^{5-7}$

The basic rate interface supports a $2 \mathrm{~B}+\mathrm{D}$ $(2 \times 64 \mathrm{~kb} / \mathrm{s}+16 \mathrm{~kb} / \mathrm{s})$ aggregate data rate, which offers approximately an order of magnitude improvement over modems and dial-up voice grade channels. Customer premise access is designated as the "user loop" (U-loop). A small unit (approximately $4 \times 7 \times 1$ in), the NT1, (AT\&T, Netherlands), terminates the U-loop and provides a standard interface to the $\mathrm{N}$-ISDN modem (terminal adapter) that is connected to the computer. The configuration and terminology are summarized in Fig 1.

Based on an image size of $1,024 \times 1,024$ pixels, 10 bits per pixel, the $2 \mathrm{~B}$ channel would support transmission of the image in approximately 80 seconds. A compression ratio of 10:1, based on the Joint Photographic Experts Group (JPEG) still image compression algorithm, ${ }^{8}$ seems to provide images of acceptable visual quality while reducing the transmission time to less than 10 seconds. Our experiments target image and information (radiology reports and hospital data) delivery to the primary care physician. Source image samples are grouped into $8 \times 8$ blocks, normalized, and transformed using a discrete cosine transform (DCT). The coefficients are then quantized and entropy encoded for transmission. Additional details are presented in the following section. Although in excess of the usual targets of user response times ( 2 seconds or less), the achievable delivery time may be satisfactory for load- 


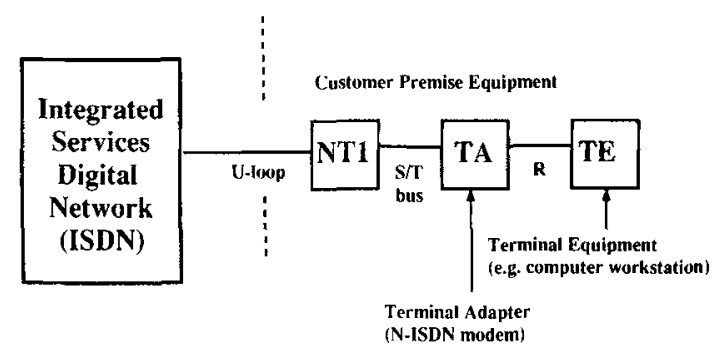

Fig 1. ISDN terminology: U loop, 2-wire circuit providing subscriber line access to the ISDN; S/T bus, 4-wire circuit that allows access to the individual channels of the basic rate interface; NT1, network termination unit that provides for the physical termination of the U-loop; and R (reference point). denotes data terminal equipment with differing communication technologies (eg, V.35, RS 232, Ethernet).

ing a remote cache or low-volume viewing, given the reasonable cost (approximately $\$ 60$ per month for the service ${ }^{9}$ and less than $\$ 350$ for the computer modem ${ }^{10}$ ) and the near-term availability of the N-ISDN service. Some RBHCs have proposed usage-dependent line charges that may limit market acceptability. Our demonstration has been supported in the context of the Radiology Information and Image Management (RIM) Testbed ${ }^{11}$ at the Mallinckrodt Institute of Radiology. Digital image acquisition from both computed radiography (CR) and filmscanned projection radiographs, access to patient demographics, exam information, and electronically stored radiology reports provide an environment for developing and testing inquiry and display applications. Image databases support both clinical and basic research. The addition of a N-ISDN gateway supports geographically remote inquiry and display as shown in Fig. 2.

The testbed uses local area network technology based on Ethernet (IEEE 802) for image acquisition and database queries. Image deliv-

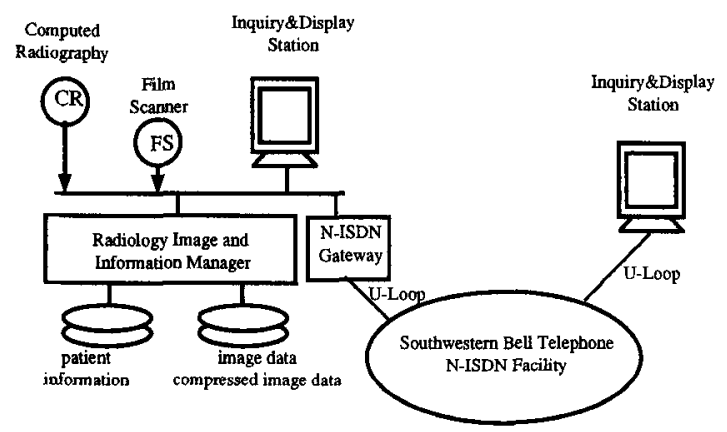

Fig 2. N-ISDN teleradiology extension to RIM. ery is supported by both Ethernet and highspeed fiber networks. The RIM is based on a local design implemented on a VAX-8530 cluster (Digital Equipment Corp, Maynard, MA). The image database implementation uses a commercial relational database product. The testbed is configured to support integration of new imaging modalities, soft-copy viewing experiments and network experimentation. The $\mathrm{N}$ ISDN gateway is implemented on a MicroVAX II using DECnet software for routing. Commercially available interface cards for the Q-Bus (DEC DIV-32, Digital Equipment Corp) support the basic rate $2 \mathrm{~B}$ channel configuration. Inquiry and Display stations have been developed using window-based software on computer workstations to support the user interface and auxiliary displays to support the display of the medical image.

\section{N-ISDN DEMONSTRATION CONFIGURATION}

\section{Workstation Configuration}

A specific implementation of our Inquiry \& Display Station, used in the N-ISDN demonstrations, is referred to as a medical doctor's workstation (MDWS). The implementation takes advantage of readily available hardware and software products, recognizing the size and cost limitation posed by the existing devices. A DEC 3520 workstation was chosen as the computer to support the N-ISDN connectivity (DECnet over the basic rate channel); the decompression of the image data is achieved via the embedded digital signal processor equipment supported by the NeXT computer (NeXT Inc, Redwood City, $\mathrm{CA}$ ) and the image display is provided by an Imlogix display unit (Imlogix, St Louis, MO). A block diagram showing the connectivity and major components of a remote MDWS Inquiry \& Display Station is shown in Fig 3.

\section{Connectivity}

The basic rate service $(2 \mathrm{~B}+\mathrm{D})$ provides a full duplex channel by time division multiplexing both user information and signaling into a common stream, yielding an aggregate of 192 $\mathrm{kb} / \mathrm{s}(2 \mathrm{~B}+\mathrm{D}=144 \mathrm{~kb} / \mathrm{s}$ plus $48 \mathrm{~kb} / \mathrm{s}$ signaling). The U loop uses a two-wire circuit for both transmit and receive. U-loop extensions between the AT\&T 5ESS switch (AT\&T, Oklahoma City, OK) located at the Advanced Tech- 


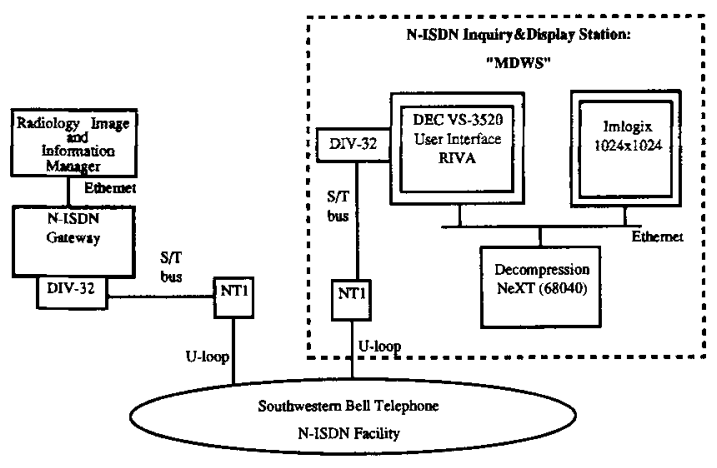

Fig 3. Block diagram of the connectivity and primary components of the $\mathrm{N}$-ISDN experimental configuration.

nology Laboratory and the demonstration sites at the Electronic Radiology Laboratory and the Technology Resources Incorporated Laboratory have been supported by T1 and D4 Channel Banks equipped with AT\&T basic rate interface transmission extension channel units. The topology is shown in Fig 4. Our U loops are supported by twisted-pair circuits of less than $200 \mathrm{ft}$ and the network termination units are located within 50 feet of the DIV-32 interface cards (Digital Equipment Corp). The DEC based N-ISDN Inquiry \& Display Station and router configuration was tested and found to support transfer rates of $120 \mathrm{~kb} / \mathrm{s}$ for two $B$ channels. ${ }^{6}$

\section{JPEG Compression/Decompression}

As shown in Fig 5, image pixels are grouped into $8 \times 8$ blocks, a simple "DC offset" is subtracted from each pixel value, and a DCT is applied to each block. A psychovisual threshold matrix (such as specified by CCIR-601 ${ }^{12}$ ) is used by the quantizer to reduce the dynamic range of the set of 64 coefficients. The matrix controls the overall data rate and image quality. Many high frequency coefficients are reduced to zero after this quantization step. A zig-zag scan orders each block of 64 coefficients from the lowest frequency (DC) to the highest frequency.

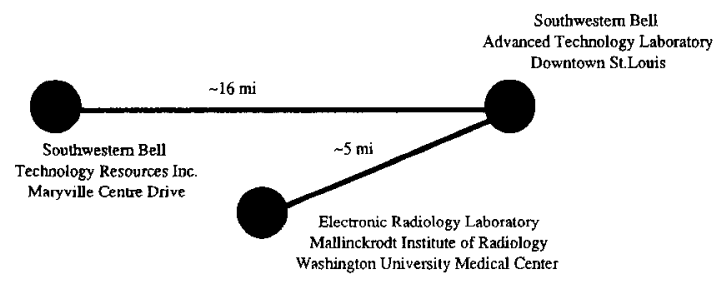

Fig 4. N-ISDN demonstration topology-St. Louis.

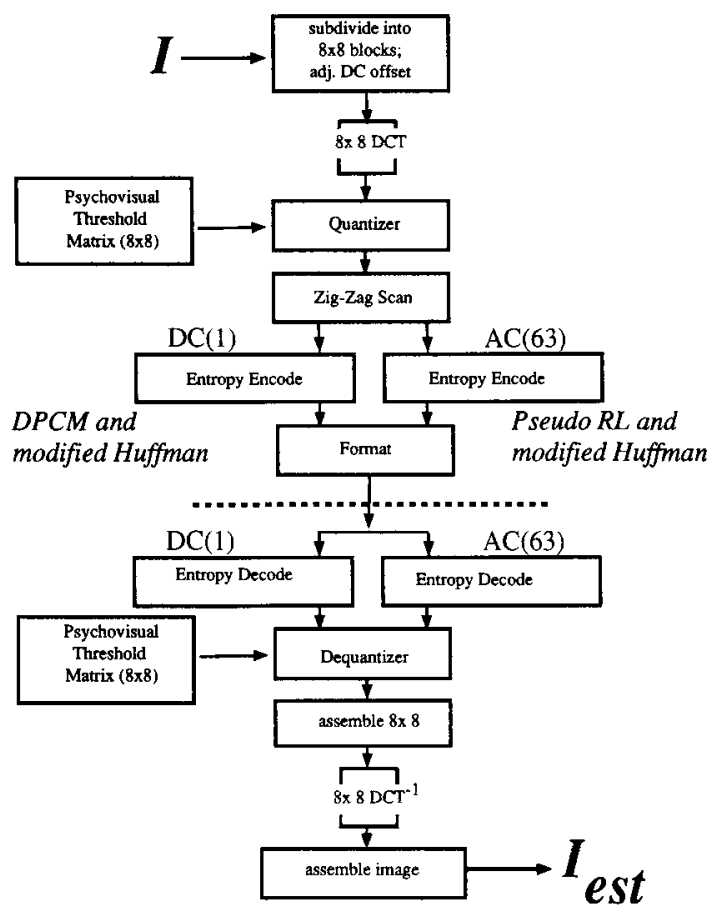

Fig 5. JPEG encoding and decoding steps.

DC coefficients are coded as the difference between the current $\mathrm{DC}$ value and the prior value. "AC coefficients" (those other than DC) are coded in tuples according to the number of zero AC coefficients before the next nonzero coefficient. A modified Huffman coding scheme is used to encode the DC and AC streams separately. The decompression of an image is accomplished by transferring (via EthernetTCP/IP) the composite stream to the NeXT computer memory, loading the decoding algorithm into the digital signal processor (DSP), activating the decoding operation, assembling the decompressed image data into NeXT memory, and, finally, transferring the image to the Imlogix display via Ethernet. A model of the decoding algorithm implementation labeled with timing results is shown in Fig 6.

\section{APPLICATION-LEVEL MEASUREMENTS AND OBSERVATIONS}

The inquiry and display application (Radiology Image Viewing Application) executes on the VAXstation 3520 (VMS, DECwindows) as shown in Fig 3. A connection (via DECnet) is established between the 3520 and the RIM system to support the queries and retrieval of compressed image data. The demonstration has 


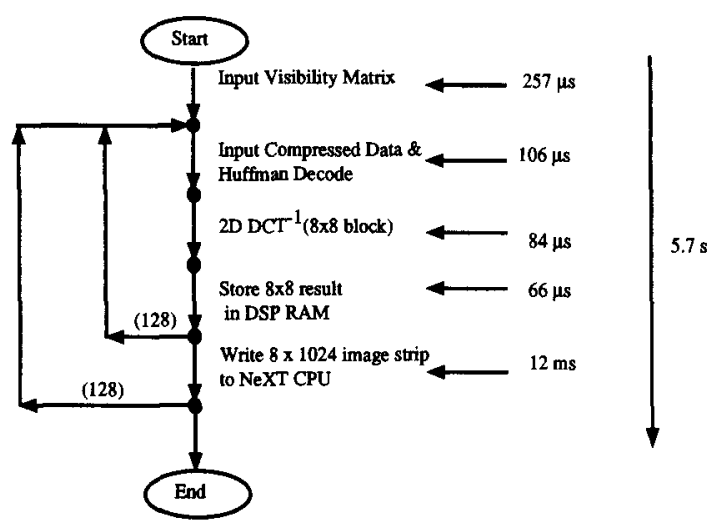

Fig 6. Signal flow model and example timing of JPEG decoding algorithm.

focused on providing a spatially compressed version $(1,024 \times 1,024)$ of $C R$ images $(2,139 \times$ 1,740 pixels $\times 10 \mathrm{bits} /$ pixel). On-line access to the related examination information and the signed radiology report augments the image data. A nominal JPEG compression ratio of 10:1 applied to the spatially compressed image results in an overall data reduction of 40:1.

Initiation of display requests by the remote MDWS are relayed to the RIM. The display request, in turn, requires a number of database queries followed by a data retrieval from a disk-based storage unit. The "compressed image data" is then transmitted through the DECnet router, transmitted via ISDN to the DEC VAXstation, and retransmitted via the remote Ethernet segment to the NeXT computer via a TCP/IP connection. Decompression is accomplished with the NeXT's digital signal processor environment and the image pixels are reassembled in the NeXT's memory. Finally, the image is transmitted over the remote Ethernet segment to the Imlogix display unit. The aggregate time for this set of operations was 28 seconds. A degree of pipelining was implemented as shown in Fig 7. The elapsed time from image request to completed display is less than 20 seconds.

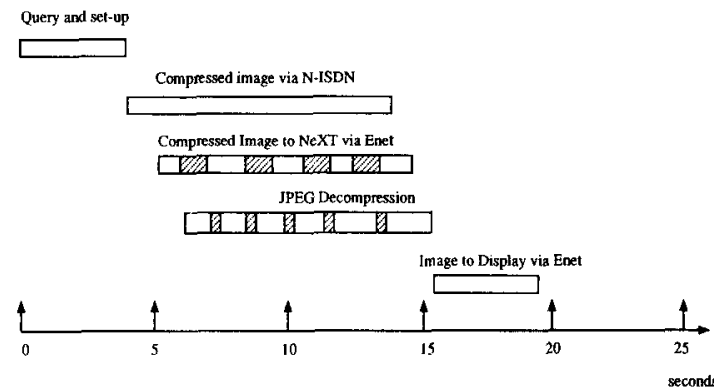

Fig 7. Example of inquiry and display elapsed time measurement.

Additional opportunities to improve the response time include reduction of the query and setup time, direct memory access transfers between the DSP and the NeXT 68040 processor, multithreaded coding on the NeXT, and moving the image data to the display as each iteration of the outer loop (Fig 6) is completed. Even with an optimized compression system, the lower bound for retrieval for compressed CR images is limited by the N-ISDN transmission time and is on the order of 9 seconds. The combination of JPEG and the N-ISDN channels offers opportunities for modest cost and ubiquitous accessibility to both radiology reports and images.

\section{ACKNOWLEDGMENTS}

The authors acknowledge the support for this project provided by Southwestern Bell Technology Resources, Inc, and Southwestern Bell Telephone Company, St Louis, MO. The development of RIM and many aspects of the inquiry and display stations were funded by Mallinckrodt Institute of Radiology through the continued support of its Director, Ronald Evens, MD, and the chief of Diagnostic Radiology, R Gilbert Jost, MD. Digital Equipment Corporation, Maynard, MA and Imlogix, St Louis, MO, provided equipment and technical support for the RIM testbed. Design, implementation, and testing of the decompression algorithm on the NeXT's embedded digital signal processor were contributed by Evren Senol and Robert C. Lewis. Special thanks to Steven Wimmer and Tom Leith of ERL for their contribution in the development of RIM and support of the demonstrations.

\section{REFERENCES}

1. Taff A: Carriers, vendors vow to unite on ISDN. Network World 8:11-12, 1991

2. Garfinkel SI: ISDN-kit launches NeXT into communications future. NeXT World 2(1):18, 1992

3. North American ISDN User's Forum and the Corporation for Open Systems ISDN Executive Council: Transcontinental ISDN Project for 1992, November 16-20, 1992
4. Cox JR, Blaine GJ, Dubetz MW, et al: A demonstration of medical communications based on a ATM broadband network technology. Presented at SPIE Medical Imaging V, 1645-05, Newport Beach, CA, February 1992

5. Cox JR, Moore SM, Blaine GJ, et al: Optimization of trade-offs in error-free image transmission. Proceedings of SPIE Medical Imaging III: Image Capture and Display, Vol 1091, pp 19-30, 1989 
6. Blaine GJ, Ferguson RC, Studt JW, et al: ISDN: Early experiments as a wide-area extension to LAN-based PACS. Proceedings of SPIE Medical Imaging IV: PACS System Design and Evaluation, Vol 1234, pp 140-146, 1990

7. Baxter KG, Wetzel LH, Murphey MD, et al: Wide area networks for teleradiology. J Digi Imag 4:51-59, 1991

8. Wallace GK: The JPEG still picture compression standard. Communications of the ACM 34:30-44, 1991

9. Southwestern Bell Corp, St Louis, MO, personal communication (Interim Texas Tariff for locations served directly from ISDN switch)

10. Garfinkel SI: ISDN comes of age. NeXT World 2:43-45, 1992

11. Wimmer MS, Blaine GJ, Jost RG, et al: A distributed approach to integrated inquiry and display for radiology. $\mathrm{J}$ Med Syst 15:299-309, 1991

12. Encoding parameters of digital television for studies: CCIR Recommendation CC1R-601 